\title{
Artigo/Article
}

\section{Mielopatia associada ao HTLV-1: análise clínico-epidemiológica em uma série de casos de 10 anos}

\author{
HTLV-1 associated myelopathy: clinical and epidemiological profile in a 10-year case \\ series study
}

\author{
Ana Paula Silva Champs ${ }^{1,3}$, Valéria Maria de Azeredo Passos ${ }^{2,3}$, Sandhi Maria Barreto ${ }^{3,4}$, Luiz Sergio Vaz \\ e João Gabriel Ramos Ribas ${ }^{1}$
}

\begin{abstract}
RESUMO
Introdução: A mielopatia associada ao retrovírus HTLV-1 (HAM/TSP) é uma doença progressiva e incapacitante. O objetivo deste trabalho é determinar características clínicoepidemiológicas de pacientes com HAM/TSP. Métodos: Série de casos admitidos de 01/1998 a 12/ 2007, em hospital de reabilitação utilizando os critérios diagnósticos de HAM/TSP. Resultados: Participaram 206 pacientes, dos quais, 67\% eram mulheres, com 53 anos de média de idade, nove anos de média de duração de doença. Os sintomas mais frequentes foram a diminuição da força em membros inferiores, espasticidade, dor, presença de bexiga neurogênica e a constipação intestinal. Os sinais neurológicos foram hiperreflexia, Babinsky, Hoffmann e neuropatia periférica. A presença de dor, de espasticidade muscular e de atrofia medular à ressonância nuclear magnética de medula espinhal foram associadas à duração da doença $(p<0,05)$. Conclusões: A HAM/TSP é uma doença de curso incapacitante e progressiva, em que a dor é relatada precocemente, enquanto a atrofia medular torácica e a espasticidade surgem em fase mais tardia. Existem casos de HAM/TSP com provável transmissão do vírus por via vertical.
\end{abstract}

Palavras-chaves: Epidemiologia. Vírus linfotrófico de células T humana tipo 1. Paraparesia espástica tropical.

\begin{abstract}
Introduction: Human T cell lymphotropic virus type 1 (HTLV-I) myelopathy (HAM/TSP) is a progressive disabling disorder. This work aimed to analyze clinical features and epidemiology in a sample of HAM/TSP. Methods: All HTLV-1 infected patients with diagnostic criteria for HAM/TSP, consecutively admitted to the Sarah Hospital from 1998 to 2007, were included in the study. Results: 206 patients (67\% females; mean age: 53.8 years-old) were diagnosed with HAM/TSP. The mean time of evolution was 9.0 years. The most common neurological symptoms were chronic progressive spastic paraparesis, spasticity, pain, neurogenic bladder and neurogenic bowel. The neurological findings were hyperreflexia, Babinsky, Hoffman and peripheral neuropathy. Pain, spasticity and spinal cord atrophy, observed in MRI, were associated with time of disease $(\mathrm{p}<0.05)$. Conclusions: HAM/TSP is a very disabling disorder, in which pain is reported early, while spasticity and thoracic spinal cord atrophy appear in a later phase of the disease. Cases of HAM/TSP exist with a probable vertical viral transmission.
\end{abstract}

Key-words: Epidemiology. Human T cell lymphotropic virus 1. Tropical spastic paraparesis.

1. Rede Sarah de Hospitais de Reabilitação, Unidade Belo Horizonte, Belo Horizonte, MG. 2. Faculdade de Medicina, Departamento de Clínica Médica, Universidade Federal de Minas Gerais, Belo Horizonte, MG. 3. Programa de Pós-Graduação em Ciências da Saúde do Adulto, Faculdade de Medicina, Universidade Federal de Minas Gerais, Belo Horizonte, MG. 4. Faculdade de Medicina, Departamento de Medicina Preventiva e Social, Universidade Federal de Minas Gerais, Belo Horizonte, MG. Endereço para correspondência: Dra. Ana Paula Silva Champs. Hospital Sarah. Av. Amazonas 5953, Gameleira, 30510-000 Belo Horizonte, MG.

Tel: $55313379-2600$

e-mail: anachamps@yahoo.com.br

Recebido para publicação em 08/02/2010

Aceito em 20/05/2010

\section{INTRODUÇÃO}

O Human T cell Lymphotropic virus (HTLV) é um vírus que pertence à família retroviridae e, atualmente são descritos quatro tipos: HTLV-1, HTLV-2, HTLV-3 e HTLV-4. O HTLV-1 é o tipo viral mais associado a doenças. Foi descrito em $1977^{1}$, associado ao linfoma de células T e à mielopatia. O HTLV-2 foi identificado em 1982 e é associado a raros casos neurológicos ${ }^{2}$.

A mielopatia é a manifestação neurológica mais frequente do HTLV-1. Entretanto, casos de uma paraparesia espástica idiopática foram descritos muitos anos antes do HTLV-1 ser isolado. Em 1969, o termo paraparesia espástica tropical (TSP) foi usado para descrever três casos similares ${ }^{3}$. A associação da paraparesia espástica tropical com o HTLV-1 foi reconhecida na década de $80^{4,5}$. Em 1986, Osame cols ${ }^{4}$ sugeriram que o termo tropical era inadequado, pelo fato da doença ser endêmica em uma região temperada (Japão), passando a denominá-la mielopatia pelo HTLV-1 (HAM). No Brasil, em 1989, Martins-Castro cols ${ }^{6}$ relataram a presença de anticorpos HTLV-1 em 37,5\% dos casos de mielopatia de etiologia indeterminada. Desde então, a mielopatia associada a $\mathrm{HTLV}-1$ /paraparesia espástica tropical (HAM/TSP) tem sido descrita de forma disseminada em várias regiões do mundo ${ }^{7}$.

A presença do HTLV-1 no paciente não necessariamente gera manifestações patológicas no portador. Diferentes fatores na interação vírus/hospedeiro/ambiente determinam o desenvolvimento da doença, que pode apresentarse como manifestação hematológica (leucemia/ linfoma de células T) ou inflamatória (mielopatia, uveíte, artrite reumatóide ou dermatite infecciosa etc). A resposta das células T CD8+ é o evento que determina o rumo da infecção. Nos pacientes sintomáticos, nota-se carga proviral elevada e resposta imune aumentada. Sabe-se que o HTLV-1 atravessa a barreira hemato-encefálica juntamente com os linfócitos infectados, principalmente os CD $4+^{8}$. 
O diagnóstico da infecção é feito quando existe suspeita clínica e na triagem sorológica em populações ${ }^{9,10}$. O paciente sintomático, habitualmente, é proveniente de clínicas de neurologia ou oftalmologia, devido à presença de mielopatia lentamente progressiva. Os portadores assintomáticos do vírus são diagnosticados quando fazem triagem em bancos de sangue e na investigação dos possíveis contatos de pacientes positivos ${ }^{9}$.

A mielopatia associada ao HTLV-1 (HAM/TSP) ocorre em mais de $4 \%$ dos seus portadores. A HAM/TSP cursa com quadro clínico caracterizado por acometimento de indivíduos predominantemente na quarta e na quinta décadas de vida, raramente, antes dos 20 anos ou após os 70 anos, com início insidioso e evolução lentamente progressiva ${ }^{11}$. Há predominância do sexo feminino sobre o masculino, em proporção de 2:1 a 3:1 $1^{12,13}$. Os distúrbios de marcha, fraqueza e enrijecimento dos membros inferiores são consequentes à diminuição gradual da força muscular e da espasticidade nos miótomos acometidos ${ }^{14-18}$. O paciente diminui gradativamente a deambulação, necessitando, ao longo do tempo, de auxílio para locomoção (bengalas e andadores) até evoluir para o uso da cadeira de rodas. $\mathrm{O}$ tempo médio de evolução descrito na literatura varia de poucos meses a várias décadas ${ }^{11,15}$.

Os sintomas de disfunção vésico-intestinal e sexual podem ser uma das primeiras queixas do paciente. Geralmente, caracteriza-se por urge-incontinência urinária, constipação intestinal e disfunção erétil na população masculina ${ }^{11,15,16}$.

No Brasil, grande parte dos trabalhos científicos descreve os aspectos clínicos e epidemiológicos de portadores assintomáticos, e há alguns artigos que se dedicam ao conhecimento da população com mielopatia associada ao HTLV-1. O presente estudo tem por objetivo uma descrição de uma série de casos dos pacientes com HAM/TSP, atendidos em um importante centro de referência público, para tratamento de mielopatias, comparar os resultados desse estudo com as demais publicações brasileiras, além de definir quais características clínicas e de imagem se associam à duração da doença.

\section{MÉTODOS}

Trata-se de um estudo tipo série de casos de pacientes com mielopatia pelo HTLV-1, acompanhados no Programa de Reabilitação de Lesão Medular Adulto da Unidade de Belo Horizonte da Rede Sarah de Hospitais de Reabilitação, daqui em diante denominado Hospital Sarah Belo Horizonte, admitidos no período de janeiro de 1998 a dezembro de 2007.

Os dados dos pacientes foram obtidos através do prontuário eletrônico. O protocolo de investigação da mielopatia não traumática, utilizado na instituição. Todos os 206 pacientes adultos (18+ anos) que atendiam aos critérios diagnósticos para HAM/TSP foram selecionados ${ }^{10}$.

A duração da doença foi definida como a diferença entre a data do início dos sintomas e a data de admissão neste serviço de reabilitação. Todos os diagnósticos clínicos seguiram diretrizes internacionais e nacionais, sendo assim a hipertensão arterial sistêmica (HAS) foi definida como $\mathrm{PA} \geq 140 / 90 \mathrm{mmHg}$ e/ou uso de anti-hipertensivo ${ }^{19}$; diabetes melito foi definido através da glicemia de jejum ou teste de tolerância ${ }^{20}$; o diagnóstico de alterações tireoidianas foi baseado nos exames laboratoriais de TSH e T4 livre ${ }^{21}$; a depressão definida de acordo com os critérios diagnósticos do DSM-IV (Diagnostic and Statistical Manual of Mental) ${ }^{22}$. As alterações vesicais foram definidas como espásticas ou flácidas, através do exame urodinâmico ${ }^{23}$. A constipação intestinal foi classificada de acordo com os critérios de intervalo de dias e aspectos das fezes ${ }^{24}$. A disfunção erétil foi definida através da avaliação etiológica (psicogênica, vascular, neurogênica, hormonal, medicamentosa) e/ou do Índice Internacional de Disfunção Erétil (IIEF) ${ }^{25}$. Foi utilizada a classificação do American Spinal Injury Association (ASIA) para estabelecer critérios de lesão neurológica, em níveis sensitivo e motor ${ }^{26}$.

\section{Análise estatística}

Após coleta, os registros foram inseridos no aplicativo Excel. A descrição dos pacientes segundo características epidemiológicas, sóciodemográficas e clínicas, foi realizada neste mesmo programa. A seguir, foi feita uma análise exploratória dos dados, para obter as características da população estudada no programa SPSS 17.0. Os Testes do Qui-quadrado e Exato de Fisher foram utilizados para comparar as proporções nas variáveis categóricas. Para as comparações entre médias e medianas, utilizou-se os testes $\mathrm{T}$ não emparelhado e de Mann-Whitney, respectivamente. $O$ valor de significância considerado foi $p<0,05$, bicaudal.

\section{Ética}

Este estudo foi aprovado pelo Comitê de Ética em Pesquisa da UFMG (parecer 664/08 COEP UFMG) e da Rede Sarah de Hospitais de Reabilitação. Por se tratar de análise de dados secundários, não foi necessária a obtenção de termo de consentimento livre e esclarecido ${ }^{27}$.

\section{RESULTADOS}

Entre os 206 pacientes, houve predomínio de mulheres (67\%), com proporção de 2:1 em relação ao sexo masculino. A média de idade dos pacientes, no início dos sintomas, foi de 44,8 $( \pm 14,1)$ anos e, no momento da admissão no serviço, de $53,8( \pm 13,1)$ anos, ou seja, um tempo médio de 9,0 $( \pm 7,8)$ anos até o início da mielopatia e a admissão no serviço de reabilitação (Tabela 1). A duração da doença variou de poucos meses a 34 anos. Foi observada baixa escolaridade entre os pacientes, sendo $72 \%$ com até 8 anos de estudo. A maioria está procedente das regiões metropolitana e central de Minas Gerais (81\%).

Com relação aos possíveis modos de transmissão do HTLV, 23 pacientes (11\%) referiram passado de transfusão sanguínea, 32 (16\%) tinham passado de doença sexualmente transmissível, sendo a mais frequente a blenorragia e apenas dois pacientes relataram uso endovenoso de drogas ilícitas. A co-infecção com HIV estava presente em quatro pacientes e houve um caso de co-infecção pelo vírus de hepatite B, outro de hepatite Ceum terceiro de HTLV-2. A presença de familiares soropositivos para o HTLV-1 foi observada em 47 pacientes (22\%), sendo que destes, 32 (68\%) dos familiares eram cônjuges e 9 (19\%) eram filhos. A sorologia materna positiva foi encontrada em 11 (23\%), sugerindo a transmissão materno-fetal. Os pacientes que têm a mãe HTLV positiva tiveram média de idade do início dos sintomas de 31,9 anos, contra 45,4 anos para os demais pacientes ( $p$ do teste bilateral de Student $<0,001$ ).

O tabagismo foi relatado em 54 (26\%) e uso diário de álcool em $24(12 \%)$ casos.

As co-morbidades mais frequentes foram a hipertensão arterial em $53(26 \%)$ e o diabetes melito em 15 (7\%) dos pacientes. A insuficiência renal crônica (IRC), complicação que pode estar relacionada à bexiga neuropática, estava presente em apenas seis (3\%) dos pacientes. A presença de HAM/TSP, associada à leucemia/ linfoma de células $\mathrm{T}$, ocorreu em dois pacientes. 
TABELA 1 - Perfil sóciodemográfico e clínico dos 206 pacientes com HAM/ TSP, atendidos de janeiro de 1998 a dezembro de 2007, no Hospital Sarah de Belo Horizonte.

\begin{tabular}{|c|c|c|}
\hline \multirow[b]{2}{*}{ Característica } & \multicolumn{2}{|c|}{ Casos } \\
\hline & $\mathrm{n}^{\mathrm{o}}$ & $\%$ \\
\hline \multicolumn{3}{|l|}{ Sexo } \\
\hline feminino & 139 & 67,0 \\
\hline masculino & 67 & 33,0 \\
\hline \multicolumn{3}{|l|}{ Idade no início dos sintomas, em anos } \\
\hline média (desvio-padrão) & \multicolumn{2}{|c|}{$44,8( \pm 14,1)$} \\
\hline mediana $\left(Q_{1}-Q_{3}\right)^{*}$ & \multicolumn{2}{|c|}{$46(35-54)$} \\
\hline \multicolumn{3}{|l|}{ Idade de admissão no serviço, em anos } \\
\hline média (desvio-padrão) & \multicolumn{2}{|c|}{$52,8( \pm 13,1)$} \\
\hline mediana $\left(Q_{1}-Q_{3}\right)^{*}$ & \multicolumn{2}{|c|}{$55(47-62)$} \\
\hline \multicolumn{3}{|l|}{ Duração da doença, em anos } \\
\hline média (desvio-padrão) & \multicolumn{2}{|c|}{$9,0( \pm 7,8)$} \\
\hline mediana $\left(Q_{1}-Q_{3}\right)^{*}$ & \multicolumn{2}{|c|}{$6,5(3,0-12,0)$} \\
\hline \multicolumn{3}{|l|}{ Escolaridade } \\
\hline analfabeto & 27 & 13,0 \\
\hline 1 a 8 anos & 148 & 72,0 \\
\hline 9 a 11 anos & 25 & 13,0 \\
\hline mais de 12 anos & 6 & 3,0 \\
\hline \multicolumn{3}{|l|}{ Procedência } \\
\hline região Central de Minas Gerais e Metropolitana de Belo Horizonte & e 166 & 80,6 \\
\hline outras regiões de Minas Gerais & 31 & 15,0 \\
\hline outros Estados do Brasil & 9 & 4,4 \\
\hline \multicolumn{3}{|l|}{ Sintomas à admissão } \\
\hline distúrbio de marcha & 157 & 76,2 \\
\hline alterações vesicais & 182 & 88,4 \\
\hline constipação intestinal & 161 & 78,0 \\
\hline dor & 104 & 50,5 \\
\hline espasticidade & 163 & 79,4 \\
\hline disfunção erétil & 39 & 18,9 \\
\hline
\end{tabular}

Os sintomas mais frequentes foram a diminuição da força em membros inferiores e consequente distúrbio de marcha, relatado por 157 dos pacientes $(76,2 \%)$, a presença de bexiga neuropática, em 182 (88,4\%), e a constipação intestinal, em 161 (78,2\%) dos casos. $\mathrm{O}$ relato de dor esteve presente na metade dos pacientes.
A dor foi caracterizada como neuropática em 65 (63\%) casos, de características osteomusculares em 35 (34\%) e mista (osteomuscular e neuropática) em quatro pacientes. Os locais mais frequentes de dor foram membros inferiores em 67 (65\%) dos indivíduos, seguido da coluna tóraco-lombar em 29 (28\%) pacientes. A dor neuropática foi identificada, principalmente, em membros inferiores em 59 (91\%) dos 65 pacientes e a dor osteomuscular foi mais frequente na coluna tóraco-lombar em 25 (71\%) dos 35 pacientes.

Cerca da metade, 99 (48\%) dos pacientes, relatou sensibilidade tátil e dolorosa preservada em todo o corpo. Naqueles com redução da sensibilidade, o nível sensitivo mais frequentemente alterado foi o torácico em 62 (30\%) pacientes. A motricidade estava diminuída em membros inferiores a partir de L2 em 117 (57\%) pacientes. A alteração de sensibilidade vibratória em membros inferiores ocorreu em 81 (39\%) dos pacientes e foi rara em membros superiores, apenas em oito (4\%) pacientes. Em 16 (8\%) casos, houve alteração de noção de posição segmentar em membros inferiores.

A hiperreflexia em membros inferiores estava presente em 185 (90\%) dos casos e a tetrahiperreflexia em 101 (49\%). Em 65 pacientes (32\%), o sinal de Hoffmann era positivo, mas apenas quatro destes, mostravam diminuição de sensibilidade em membros superiores e somente três tinham diminuição de força em membros superiores.

Com relação ao perfil laboratorial, o hemograma mostrou-se normal em 130 (63\%) dos pacientes, detectou anemia normocítica e normocrômica em 27 (13\%), leucopenia em 26 (13\%) e eosinofilia em 16 (8\%), não foi realizado em $3 \%$. Relativamente, a parasitas intestinais, a amebíase estava presente em 11 pacientes, seguida da estrongiloidíase em sete.

O ultrassom de rins e vias urinárias foi realizado em todos os pacientes na admissão, e evidenciou a presença de hidronefrose em 30 (15\%) pacientes. O estudo urodinâmico foi realizado em 161 dos pacientes, evidenciando bexiga com hiperatividade em 116 (72\%) e arreflexa em 30 (19\%) e nos demais sem alterações. Dos pacientes que apresentaram hidronefrose à admissão, 26 (86\%) apresentavam hiperatividade detrusora.

A eletroneuromiografia foi realizada em 91 pacientes. Mostrouse normal em 30 (33\%), sugeriu mielopatia em 41 (45\%), polineuropatia em 17 (19\%) e miopatia em 3 (3\%) pacientes. A polineuropatia encontrada evidenciou característica sensitiva ou sensitiva-motora axonal em membros inferiores, porém um destes pacientes com polineuropatia apresentava diabetes melito tipo II, outro apresentava antecedente patológico de hanseníase e um terceiro apresentava deficiência de vitamina $\mathrm{B} 12$, o que poderia gerar um viés de confusão.

Na admissão, 109 (53\%) pacientes não usavam auxílio locomoção, 29 (14\%) utilizavam bengala em T, 18 (9\%) uma ou duas bengalas canadenses, 14 (7\%) andador e 36 (17\%) cadeira de rodas.

A presença de dor, de espasticidade muscular, de alterações na ressonância nuclear magnética da medula espinhal e o uso de auxílio-locomoção foram associados à duração dos sintomas (Tabela 2).

Outros dados, tais como diferenças entre gêneros, idade, escolaridade, 
hiperreflexia de membros superiores, prevalência de sintomas, sinal de Hoffmann, nível neurológico segundo a ASIA, alterações de sensibilidade, alterações na eletroneuromiografia, não evidenciaram associações estatísticas significativas com a duração dos sintomas.

\section{DISCUSSÃo}

Os achados do ponto de vista clínico e sócio-demográfico estão em conformidade com os achados dos demais estudos das outras regiões (nordeste e sul) e estados brasileiros (São Paulo e Rio de Janeiro). Nota-se predominância do sexo feminino ${ }^{12}$, baixa escolaridade e o início dos sintomas por volta da quarta e quinta décadas da vida ${ }^{6,12,18}$.

Parecem existir diferenças, quando se trata dos prováveis modos de aquisição da infecção. A história da transfusão sanguínea foi menor do que o descrito anteriormente na literatura ${ }^{6,12,18}$; porém, foi considerado nesse estudo somente transfusão sanguínea recebida até dezembro de 1992, já que, em 1993 iniciou-se, no Brasil, a pesquisa anticorpos HTLV, no sangue doado. A presença de doenças sexualmente transmissíveis também não foi tão frequente quanto em estudos previamente descritos ${ }^{6,12,18}$, assim como a co-infecção com HIV, vírus de hepatite B e C, além do HTLV -2. O uso de drogas ilícitas também evidenciou índices reduzidos. No entanto, foi maior a prevalência de familiares com sorologia positiva para $\mathrm{HTLV}^{6,12,18}$. Esse achado pode estar relacionado com o fato do Hospital Sarah pesquisar rotineiramente esta sorologia em familiares.

A avaliação rotineira dos familiares levou ao achado de que, aparentemente, existem pacientes com mielopatia associada ao HTLV-1, com provável transmissão vertical (11 mães HTLV soropositivas). Este dado deve ser analisado com cautela, já que apenas um estudo filogenético dos vírus das mães e filhos, poderá confirmar esta afirmativa. Entretanto esse resultado deve ser considerado como estudo de futuras pesquisas, porque contradiz a literatura científica atual ${ }^{28}$, que afirma que pacientes que adquiriram a infecção pelo HTLV-1 por via vertical, não desenvolvem HAM/ TSP, e sim, leucemia/linfoma de células T.

Em conformidade com os achados anátomo-patológicos, descritos na literatura, a atrofia medular torácica, detectada na ressonância nuclear magnética, surge em uma fase mais tardia da evolução da doença ${ }^{17,29}$. Entretanto, ao contrário de outros autores ${ }^{6,13,18}$, no presente estudo, a espasticidade parece ser encontrada também em fase mais tardia da mielopatia, podendo significar que, no início do quadro neurológico, nem sempre a espasticidade é um achado frequente.

A relação inversa entre dor, seja com características neuropáticas e/ou osteomuscular, e duração da doença sugere que o paciente procura mais precocemente o serviço de saúde, quando ela está presente. Outra hipótese seria que, numa fase mais tardia, haveria menos receptores medulares para a dor, devido à atrofia medular. Nesse caso, necessitaria um estudo longitudinal para avaliar se a dor é modificada no curso natural da doença.

Predominam os sintomas motores caracterizados pela diminuição da força em membros inferiores e consequente distúrbio de marcha, as alterações vesicais relatadas em sua maioria como urge-incontinência e a constipação intestinal ${ }^{13,18}$.

Cerca da metade dos pacientes mantém a sensibilidade preservada em todo o corpo e o nível sensitivo mais acometido é o torácico. A tetrahiperreflexia e sinal de Hoffmann positivo são sinais frequentes, apesar do pouco acometimento da força muscular e da sensibilidade dos membros superiores, com resultado compatível com o descrito em publicações anteriores ${ }^{6,14}$. Nota-se a importante prevalência da disfunção erétil nos pacientes masculinos, problema grave que deve ser abordado ${ }^{30}$. Também, existem alterações da função sexual feminina, devido à diminuição da sensibilidade perineal e das próprias limitações físicas, da progressão da doença, entretanto, no presente estudo não foi feita uma análise destes dados por não ser uma pesquisa rotineira neste serviço de reabilitação.

A frequência da estrongiloidíase varia bastante entre os estudos e as diferentes regiões brasileiras. Alguns pesquisadores relatam a estreita relação entre fatores imunológicos nos pacientes com a infecção pelo HTLV-1 e essa parasitose ${ }^{31,32}$. Esse dado pode estar subestimado, já que neste serviço, não se faz rotineiramente o teste de Baerman-Morais, específico das larvas deste parasita.

A polineuropatia ${ }^{33}$, presente em 17 pacientes, pode estar relacionada também às co-morbidades presentes na história pregressa de alguns pacientes, tais como o diabetes melitus tipo II, a hanseníase e a hipovitaminose B12. Aparentemente, as alterações em nervos periféricos são raras nesta população específica, mas pode existir um viés de seleção, já que foram escolhidos os casos que preenchiam critérios para mielopatia. Alterações miopáticas, associadas à mielopatia, foram encontradas em três casos, o que pode sugerir a importância da investigação também de enzimas musculares em casos suspeitos na eletroneuromiografia ${ }^{34}$.

A bexiga hiperativa foi a alteração mais frequente, o que é compatível com a paraplegia espástica. É curiosa a presença da bexiga arreflexa, observada em $19 \%{ }^{35,36}$, considerando que o HAM/TSP é uma doença predominantemente espástica, sendo a hiperatividade detrussora o achado esperado. O presente estudo demonstra a importância da investigação do vírus HTLV-1 em pacientes com sintomas iniciais de urge-incontinência vesical ou retenção urinária, porque nota-se uma porcentagem importante dessa subpopulação com sintomas vesicais, sem ou com pouco distúrbio de marcha. Nesta pesquisa, na admissão, 109 (53\%) pacientes não usavam auxílio locomoção para marcha, mas 94 destes, apresentavam queixas de sintomas vesicais, cujos estudos urodinâmicos demonstraram 18 pacientes com arreflexia vesical e 55 com hiperatividade detrussora.

Este artigo contempla uma grande série de casos com HAM/ TSP, descrita em um único centro no Brasil. Uma possível limitação à interpretação dos dados é o viés de seleção, já que esses pacientes, encaminhados para um centro de referência, podem apresentar-se com os aspectos mais graves da doença.

Sabe-se que a mielopatia associada ao HTLV-1, apesar de rara, é uma doença grave, incapacitante, que cursa com elevada morbidade, com comprometimento das atividades de vida diária e da qualidade de vida.

Conhecer o perfil clínico e epidemiológico desta população pode ajudar o profissional de saúde suspeitar, mais frequentemente, do diagnóstico, estimular a investigação dos sintomas associados e solicitar exames necessários.

\section{AGRADECIMENTOS}

Valéria Maria de Azeredo Passos e Sandhi Maria Barreto, bolsistas de produtividade em pesquisa do CNPq (processos no 00908 /95 e no 300159/99-4). 


\section{CONFLITO DE INTERESSE}

Os autores declaram não haver nenhum tipo de conflito de interesse no desenvolvimento do estudo.

\section{REFERÊNCIAS}

1. Uchiyama T, Yodoi J, Sagawa K, Takatsuki K, Uchino H. Adult T-cell leukemia: clinical and hematologic features of 16 cases. Blood 1977; 50:481-492.

2. Kalyanaraman VS, Sarngadharan MG, Robert-Guroff M, Miyoshi I, Golde D, Gallo RC. A new subtype of human T-cell leukemia virus (HTLV-II) associated with a T-cell variant of hairy cell leukemia. Science 1982; 218:571-573.

3. Mani KS, Mani AJ, Montgomery RD. A spastic paraplegic syndrome in South India. J Neurol Sci 1969; 9:179-199.

4. Osame M, Usuku K, Izumo S, Ijichi N, Amitani H, Igata A, et al. HTLV-I associated myelopathy, a new clinical entity. Lancet 1986; 1:1031-1032.

5. Rodgers-Johnson P, Gajdusek DC, Morgan OS, Zaninovic V, Sarin PS, Graham DS. HTLV-I and HTLV-III antibodies and tropical spastic paraparesis. Lancet $1985 ; 2: 1247-1248$.

6. Martins-Castro LH, Chaves CJ, Callegaro D, Nóbrega JP, Scaff M. HTLV-I associated myelopathy in Brazil: a preliminary report. Arq Neuropsiquiatr $1989 ; 7: 501-502$

7. Proietti FA, Carneiro-Proietti AB, Catalan-Soares BC, Murphy EL. Global epidemiology of HTLV-I infection and associated diseases. Oncogene $2005 ; 24: 6058-6068$.

8. Costa CMC, Dom R, Carton H, Santos TJT, Andrada-Serpa MJ.Neuropathology of human and experimental TSP/HAM: a critical review. Acta Neurol Belg 2002; 102:21-29.

9. Passos VMA, Calazans FF, Carneiro-Proietti ABS. Counseling blood donors seropositive for human T-Lymphotropic virus type I and II in a developing Country. Cad Saude Publica 1998; 14:416-420.

10. Castro-Costa CM, Araújo ABQ, Barreto MM, Takayanagui OM, Sohler MP Silva ELM et al. Proposal for diagnostic criteria of tropical spastic paraparesis/ HTLV-I-associated myelopathy (TSP/HAM). AIDS Res Hum Retroviruses 2006; 22:931-935.

11. Ribas JGR, Melo GCN. Mielopatia associada ao vírus linfotrópico humano de células T do tipo 1 (HTLV-1). Rev Soc Bras Med Trop 2002; 35:377-384.

12. Moreno-Carvalho OA, Santos JI, Di Credico G. Evidence of preferential female prevalence of HTLV-I associated tropical spastic paraparesis in Bahia-Brazil. Arq Neuropsiquiatr 1992; 50:183-188.

13. Araujo AQ, Alfonso CR, Schor D, Leite AC, Andrada-Serpa MJ. Clinical and demographic features of HTLV-1 associated myelopathy/tropical spastic paraparesis (HAM/TSP) in Rio de Janeiro, Brazil. Acta Neurol Scand 1993; 88:59-62.

14. Domingues RB, Muniz MR, Pinho JR, Bassit L, Jorge ML, Alquezar AS. Human T lymphotropic virus type I-associated myelopathy/tropical spastic paraparesis in Sao Paulo, Brazil. Clin Infect Dis 1995; 20:1540-1542.

15. Araujo AQ, Andrade-Filho AS, Castro-Costa CM, Menna-Barreto M, Almeida SM. HTLV-I-associated myelopathy/tropical spastic paraparesis in Brazil: a nationwide survey. HAM/TSP Brazilian Study Group. J Acquir Immune Defic Syndr Hum Retrovirol 1998; 19:536-541.

16. Figueiroa FL, Andrade Filho AS, Carvalho ES, Brites C, Badaró R. HTLV-I associated myelopathy: clinical and epidemiological profile. Braz J Infect Dis 2000; 4:126-30.

17. Milagres AC, Jorge ML, Marchiori PE, Segurado AA. Human T cell lymphotropic virus type 1-associated myelopathy in Sao Paulo, Brazil. Epidemiologic and clinical features of a university hospital cohort. Neuroepidemiology 2002; 21:153-158

18. Moxoto I, Boa-Sorte N, Nunes C, Mota A, Dumas A, Dourado I et al. Perfil sociodemográfico, epidemiológico e comportamental de mulheres infectadas pelo HTLC-1 em Salvador-Bahia, uma área endêmica para o HTLV. Rev Soc Bras Med Trop 2007; 40: 37-41.

19. Sociedade Brasileira de Cardiologia. Diretrizes Brasileiras de Hipertensão Arterial. Arq Bras Cardiol 2007; 89:24-79.
20. Gross JL, Silveiro SP, Camargo JL, Reichelt AJ, Azevedo MJ. Diabetes Melito: Diagnóstico, classificação e avaliação do controle glicêmico. Arq Bras Endocrinol Metab 2002; 46:16-26.

21. Larsen PR, Davies TF, Schlumberger MJ, Hay ID. Thyroid physiology and diagnostic evaluation of patients with thyroid disorders. In: Larsen PR, Davies TF, Schlumberger MJ, Hay ID editors. $10^{\text {th }}$ ed. Philadelphia: Williams textbook of endocrinology. Elsevier Science; 2003. p.331-373.

22. American Psychiatric Association. Manual diagnóstico e estatístico de transtornos mentais (DSM-4) Porto Alegre: Artes Médicas; 1995.

23. Consortium for Spinal Cord Medicine. Bladder management for adults with spinal cord injury. A Clinical Practice Guideline for Health-Care Providers. J Spinal Cord Med 2006; 29:527-573

24. Consortium for Spinal Cord Medicine. Neurogenic bowel management in adults with spinal cord injury. Clinical practice guidelines; 1998. p. 248-293.

25. Rosen RC, Riley A, Wagner G, Osterloh IH, Kirkpatrick J, Mishra A The international index of erectile function (IIEF): a multidimensional scale for assessment of erectile dysfunction. Urology 1997; 49:822-830.

26. American Spinal Injury Association. International standards for neurologica classification of spinal cord injury. Chicago: IL; 2002.

27. Diretrizes e normas regulamentadoras de pesquisas envolvendo seres humanos. Resolução no 196 do Conselho Nacional de Saúde. Ministério da Saúde; 1996.

28. Osame M, Igata A, Matsumoro M, Usuku K, Izumo S, Kosaka K. HTLV-1 associated myelopathy: a report of 85 cases. Ann Neurol 1987; 22:116-117.

29. Iwasaki Y. Human T-cell leukemia virus type I Infection and chronic myelopathy. Brain Pathol 1993; 3:1-10.

30. Castro N, Oliveira P, Freitas D, Rodrigues W, Muniz A, Carvalho E. Erectile dysfunction and HTLV-I infection: a silent problem. Int J Impot Res 2005 ; $17: 364-369$.

31. Carvalho EM, Fonseca PA. Epidemiological and clinical interaction between HTLV-1 and Strongyloides stercoralis. Parasite Immunol 2004; 26:487-497.

32. Lambertucci JR, Leao FC, Barbosa AJ. Gastric strongyloidiasis and infection by the human T cell lymphotropic virus type 1 (HTLV-1). Rev Soc Bras Med Trop 2003; 36:541-542.

33. Arakawa K, Umezaki H, Noda S, Itoh H. Chronic polyradiculoneuropathy associated with human T-cell lymphotropic virus type I infection. J Neurol Neurosurg Psychiatry 1990; 53:358-359.

34. Matsuura E, Umehara F, Nose H, Higuchi I, Matsuoka E, Izumi K. Inclusion body myositis associated with human T-lymphotropic virus-type I infection: eleven patients from an endemic area in Japan. J Neuropathol Exp Neurol 2008; 67:41-49.

35. Rocha PN, Rehem AP, Santana JF, Castro N, Muniz AL, Salgado K. The cause of urinary symptoms among Human T Lymphotropic Virus Type I (HLTV-I) infected patients: a cross sectional study. BMC Infect Dis 2007; 7:15.

36. Imamura A: Studies on neurogenic bladder due to human T-lymphotropic virus type-I associated myelopathy (HAM). Nippon Hinyokika Gakkai Zasshi 1994; 85:1106-1115 